\title{
DINÂMICA AGRÍCOLA NO DISTRITO DE CHIBUTO - MOÇAMBIQUE: UM OLHAR SOBRE O NÍVEL DE UTILIZAÇÃO DE SEMENTES CERTIFICADAS
}

\author{
António da Piedade Melo ${ }^{1}$ \\ Márcio Daniel Sitoe ${ }^{2}$ \\ Benedito Luciano Rassude ${ }^{3}$ \\ Célia das Eiras Dgedge Melo²
}

\section{Resumo}

As projeções de crescimento populacional exigem resposta dinâmica na produção de alimentos. Deste modo, reformas devem ser feitas visando à transformação dos sistemas tradicionais para sistemas baseados em tecnologias mais produtivas, que incluem, dentre várias, o uso de sementes certificadas e mais produtivas que as tradicionais. O trabalho foi realizado no Distrito de Chibuto, e teve como objetivo obter informações sobre a dinâmica na adoção e uso de sementes melhoradas por parte dos agricultores locais. Para o efeito, foram realizadas entrevistas semiestruturadas envolvendo 35 agricultores. Constatou-se que mais de metade $(62,9 \%)$ dos agricultores inqueridos utiliza sementes certificadas. Há necessidade de se acelerar o associativismo $(11,43 \%)$ de forma que a assistência aos agricultores por parte de organizações públicas e privadas, possa abrangir mais famílias. $40 \%$ dos que tem acesso as sementes certificadas, mostram-se satisfeitos com os aumentos de produtividade.

Palavras-chave: Dinâmica agrícola, pacotes tecnológicos, produção e produtividade agrícola, insumos agrícolas.

\section{AGRICULTURAL DYNAMICS IN THE DISTRICT OF CHIBUTO - MOZAMBIQUE: A VIEW ON THE LEVEL OF USE OF CERTIFIED SEEDS}

\begin{abstract}
Population growth projections require a dynamic response in food production. In this way, reforms must be made to transform traditional systems that are less productive to systems based on more productive technologies, which include, among many, the use of improved seeds. This work, carried out in the Chibuto District, aimed to obtain information on the dynamics of adoption and use of improved seeds by local farmers. For this purpose, semistructured interviews were conducted involving 35 farmers. It was found that more than half

\footnotetext{
${ }^{1}$ Docente do Departamento da Agricultura na Escola Superior de Negócios e Empreendedorismo de Chibuto da Universidade Eduardo Mondlane/Moçambique-África. Doutorado em andamento em Fitotecnia (Produção Vegetal) pela Universidade Federal de Viçosa. E-mail: antoniomelomz@gmail.com.

${ }^{22}$ Docente do Departamento da Agricultura na Escola Superior de Negócios e Empreendedorismo de Chibuto da Universidade Eduardo Mondlane/Moçambique-África.

${ }^{3}$ Licenciado em Agronegócios na Escola Superior de Negócios e Empreendedorismo de Chibuto da Universidade Eduardo Mondlane/Moçambique-África.
}

Sociedade e Território - Natal. Vol. 29, N. 2, p. 100-114, Jul./Dez. de 2017. 
$(62.9 \%)$ of the farmers in question use certified seed. It was verified the need to accelerate associativism $(11.43 \%)$ in order to boost the assistance to farmers by public and private organizations. $40 \%$ of those who have access to improved seeds are satisfied with increases in productivity.

Keywords: Agricultural dynamics, technological packages, agricultural production and productivity, agricultural inputs.

\section{DINÁMICA AGRÍCOLA EN EL DISTRITO DE CHIBUTO - MOZAMBIQUE: UNA MIRADA SOBRE EL NIVEL DE UTILIZACIÓN DE SEMILLAS CERTIFICADAS}

\section{Resumen}

Las proyecciones de crecimiento poblacional exigen respuesta dinámica en la producción de alimentos. Así, reformas deben ser realizadas objetivando la transformación de los sistemas tradicionales poco productivos para sistemas basados en tecnologías más productivas, que incluyen, entre otras, el uso de semillas mejoradas. El trabajo fue realizado en el Distrito de Chibuto y tuvo como objetivo obtener información sobre la dinámica en la adopción y uso de semillas mejoradas por parte de los agricultores locales. Para tal efecto, fueron realizadas entrevistas semi-estructuradas involucrando 35 agricultores. Se constató que más de la mitad $(62,9 \%)$ de los agricultores entrevistados utilizan semillas certificadas. Hay necesidad de acelerar la asociatividad $(11,43 \%)$ de forma que se dinamice la asistencia a los agricultores por parte de organizaciones públicas y privadas. $40 \%$ de los que tienen acceso a semillas mejoradas, se muestran satisfechos con los aumentos de productividad.

Palabras clave: Dinámica agrícola, paquetes tecnológicos, producción y productividad agrícola, insumos agrícolas.

\section{INTRODUÇÃO}

Moçambique é um país situado na costa sudeste de África. Com cerca de 27 milhões de habitantes, dois terços da sua população vive na zona rural contudo, a guerra civil terminada em 1992 incitou uma migração elevada para as zonas urbanas (Prakash, 2011). Á semelhança do país, o Distrito de Chibuto, possui uma população jovem ${ }^{4}$ e de matriz rural (taxa de urbanização de 28\%) (MAE, 2005).

A agricultura constitui a principal atividade produtiva praticada pela população rural moçambicana, maioritariamente sob regime de sequeiro, em explorações inferiores a 2 hectares, cujo principal objectivo é o sustento das famílias (CABRAL et al., 2016; DUCROT, 2016; ZANELLA e MILHORANCE, 2016). Ao longo de décadas, a injecção de capital

\footnotetext{
${ }^{4} 43 \%$ abaixo dos 15 anos em 2005.
}

Sociedade e Território - Natal. Vol. 29, N. 2, p. 100-114, Jul./Dez. de 2017. 
agrário, comercial e de extração de recursos naturais no meio rural, impulsionou transformações nos sistemas de produção tradicionais (DUCROT, 2017; WHITFIELD et al., 2016; ZANELLA \& MILHORANCE, 2016; MOSCA, 2014).

Os níveis de pobreza continuam elevados desde a independência de Moçambique em 1975, apesar das injecções de capital. O sucesso dos investimentos depende paralelamente de mudanças na visão dos agricultores para responderem de forma dinâmica e racional a exposição a novas tecnologias (GUANZIROLI \& GUANZIROLI, 2015; MOSCA, 2014; CUNGUARA \& DARNHOFER, 2011), garantindo disponibilidade de alimentos para consumo familiar e comércio, pelo menos no período entre Safras.

A dinâmica das práticas agrícolas tradicionais deve permitir uma rápida adoção de tecnologias com impato no aumento da produção e produtividade (CARTER, CHENG \& SARRIS, 2016; ABATE et al., 2016; MICHEELS \& NOLAN, 2016; YAMANO et al., 2016).

A evolução dos níveis de adoção e uso de sementes certificadas vem ocorrendo em taxas consideradas baixas, principalmente em países em vias de desenvolvimento, sendo comum o uso de sementes provenientes da colheita anterior, conservadas em condições que por vezes comprometem a qualidade fisiológica e sanitária, o que por sua vez compromete a produtividade agrícola (DERWISCH et al., 2016; BEYENE \& KASSIE, 2015; BEZU et al., 2014; MATHENGE, SMALE \& OLWANDE, 2014).

Parte considerável das hortícolas disponíveis nos mercados do Distrito de Chibuto, provém do Distrito de Chókwè, o que mostra a fraca capacidade de suprimento interno, que pode estar em parte relacionada ao fraco uso de tecnologias melhoradas.

Por tecnologias melhoradas entende-se o uso da mecanização agricola, sementes melhoradas, metodos de irrigação adequados, fertilizantes e pesticidas, combinados com tecnologias adequadas de conservação ambiental e mão de obra, com vista ao aumento da produção e produtividade (Cunguara \& Kelly 2015).

De acordo com Uaiene (2006) a adoção de tecnologias melhoradas em Moçambique de forma geral e Chibuto em particular, está abaixo do nível ótimo, devido a baixa renda dos produtores rurais, excassez de financiamento rural e a ineficiência da rede de comércio em levar as sementes para junto dos agricultores. Segundo Massingue et. al, (2001), apenas 5 a $10 \%$ de toda a semente utilizada pelos produtores moçambicanos é certificada. Contudo, o pacote tecnológico utilizado pelos agricultores de Chibuto, inclui o uso de tratores, arados, grades, pesticidas, fertilizantes e rega por gravidade. 
A produção de sementes certificadas em Moçambique é da responsabilidade das empresas Sementes de Moçambique (SEMOC) e PANNAR Seed Company, sendo comercializadas a grosso e a retalho. A certificação é feita pela Autoridade Nacional de Sementes, através dos Laboratórios Centrais e Regionais de Sementes (BR, 2013).

É neste contexto que com este trabalho, realizado nos Postos Administrativos de Malehice e Chaimite no Distrito de Chibuto, objetivou-se obter informação sobre a dinâmica na adoção e uso de sementes certificadas por parte dos agricultores locais.

\section{MATERIAL E MÉTODOS}

A operacionalização deste trabalho teve início com a realização de uma pesquisa exploratória, baseada na revisão bibliográfica. Seguiu-se a consumação de entrevistas semiestruturadas ${ }^{5}$.

Os Postos Administrativos (PA) em estudo possuem um total de 350 agricultores, divididos em 150 do PA Malehice e 200 do PA Chaimite, destacados na figura 01. Na amostra $^{6}$ considerou-se $10 \%$ da população, tendo sido estratificada para que em cada PA, $10 \%$ da população fizesse parte da amostra. Assim, a amostra foi de 35 agricultores, dos quais, 20 pertencentes ao Posto Administrativo de Chaimite e 15 ao Posto Administrativo de Malehice. A opção pelos locais de estudo supracitados, levou em consideração os poucos recursos disponíveis, dada a proximidade destes ao Posto Administrativo Sede ${ }^{7}$.

Dos 15 agricultores entrevistados no Posto Administrativo de Malehice, 2 pertencem a associação Tsica Ulolo, sendo os outros 13, não associados. Já no Posto Administrativo de Chaimite, 2 dos 20 entrevistados são associados, pertencendo a associação Kutxela, sendo os demais não associados.

Para a recolha de dados foi usada a técnica de amostragem probabilística estratificada $^{8}$ em dois postos administrativos onde foram realizados os estudos. Os elementos

\footnotetext{
5 Tem como característica questionamentos básicos que são apoiados em teorias e hipóteses que se relacionam ao tema da pesquisa.

${ }^{6}$ Parte da população.

${ }^{7}$ Local de trabalho dos pesquisadores.

${ }^{8}$ Consiste em dividir toda a população ou o "objeto de estudo" em diferentes subgrupos ou estratos diferentes, de maneira que um indivíduo pode fazer parte apenas de um único estrato.
}

Sociedade e Território - Natal. Vol. 29, N. 2, p. 100-114, Jul./Dez. de 2017. 
da população foram divididos em grupos não sobrepostos, o que facilitou a escolha, por meio da amostragem aleatória simples ${ }^{9}$ dentro de cada grupo.

\section{Figura 01: Mapa da Província de Gaza}

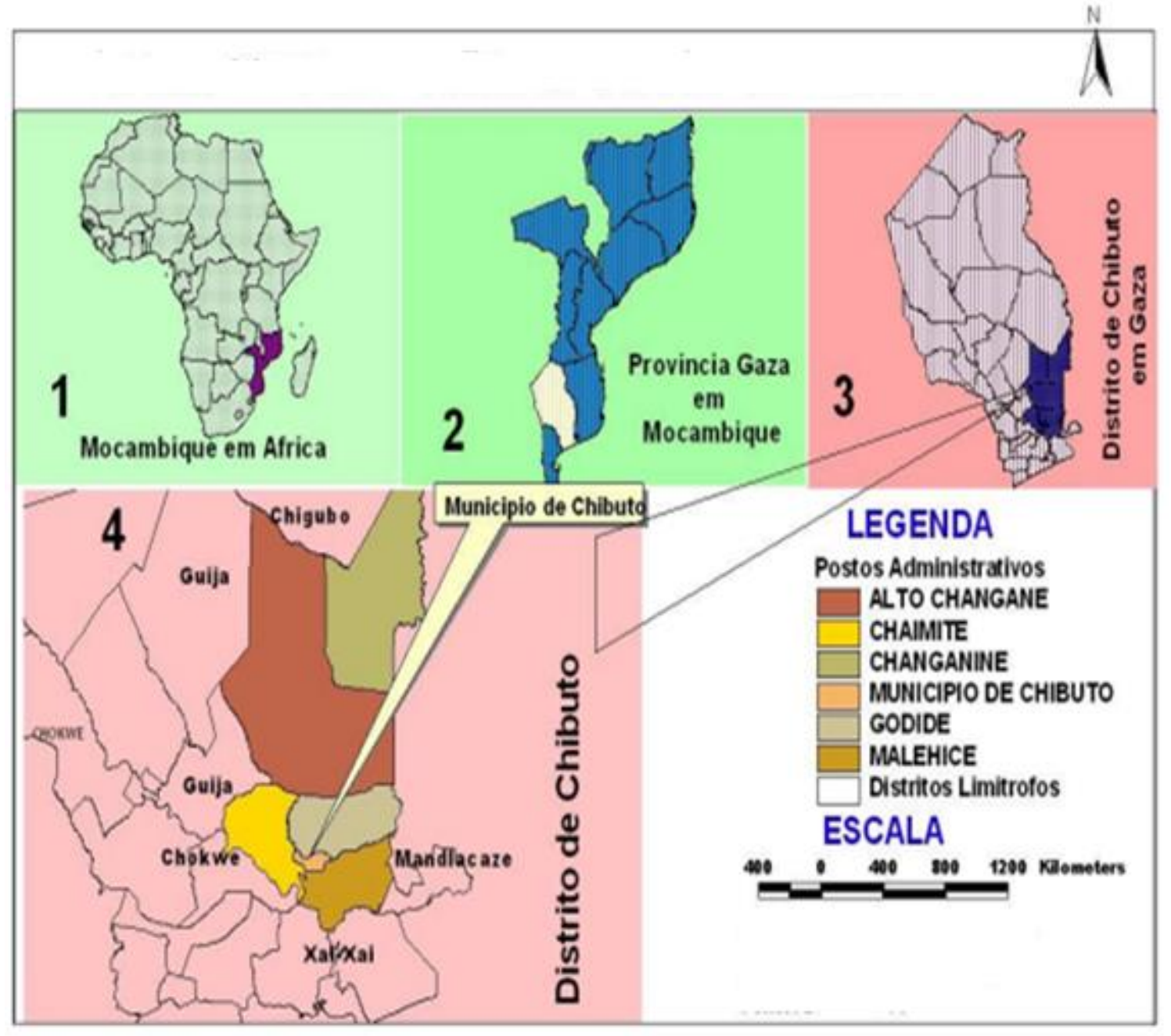

Fonte: Portal do Governo de Moçambique

https://coracaoafricano2532014.wordpress.com/2015/05/11/gaza-mocambique/

As entrevistas foram realizadas entre os dias 15 e 25 de Março de 2016, com interrupção no sábado e Domingo, com início as 6 horas e 30 minutos e término as 11 horas, tendo sido entrevistados 5 agricultores por dia.

A análise dos dados foi feita por meio de estatística descritiva ${ }^{10}$, com o auxílio do Pacote Estatístico SPSS for Windows, versão 20. As variáveis analisadas foram: Produção e produtividade de hortícolas, acesso a sementes melhoradas, conhecimento dos pacotes tecnológicos associados as sementes melhoradas.

\footnotetext{
${ }^{9}$ Técnica de amostragem onde todos os elementos que compõem o universo e estão descritos no marco amostral têm idêntica probabilidade de serem selecionados para a amostra.

${ }^{10}$ Ramo da estatística que aplica várias técnicas para descrever e sumarizar um conjunto de dados.
}

Sociedade e Território - Natal. Vol. 29, N. 2, p. 100-114, Jul./Dez. de 2017. 


\section{RESULTADOS}

A agricultura nos Postos Administrativos de Chaimite e de Malehice é maioritariamente praticada por mulheres $(74,3 \%)$ com mais de 40 anos de idade $(60 \%)$, sem o ensino primário do $1^{\circ}$ grau concluído (60\%). Exploram áreas de produção não superiores a 1 ha $(88,6 \%)$, 3 ha $(8,6 \%)$ e a 5 ha $(2,9 \%)$. De forma geral, a produção em áreas não superiores a 1 ha é praticada na sua maioria pelas mulheres para o consumo familiar e realizam a sementeira das hortícolas como tomate, cebola, cenoura e alface, sem o uso do trator para a prpeparação da área de produção. O cultivo é realizado na época fresca ${ }^{11}$, havendo contudo, os que preferem semear em períodos quentes ${ }^{12}$. Já em áreas superiores a 3 ha, na qual os homens predominam, são cultivados na maioria das vezes o tomate e o milho intercalados nas épocas fresca e quente respetivamente. Os cultivos são realizados com o uso de tratores e sistemas de irrigação, e a produção é destinada a venda.

O nível de uso de sementes certificadas é baixo $(62,9 \%)$ entre os entrevistados, tal como evidenciado na tabela 01 . No entanto, $40 \%$ mostram-se satisfeitos com os resultados visto que verificam aumento de produtividade, ao passo que $20 \%$ não tem certeza, pois observam resultados satisfatórios apenas quando chove, no caso contrário, a produção é baixa (Tabela 02). Contudo, 8,6\% dos agricultores afirmaram estar insatisfeitos pelos resultados e $31,4 \%$ se mostraram indiferentes por não utilizarem sementes certificadas na produção de hortícolas.

Tabela 01: Uso de sementes certificadas pelos agricultores do PA Malehice e do PA Chaimite

\begin{tabular}{lcc} 
AGRICULTORES QUE UTILIZAM & FREQUENCIA & PERCENTAGEM \\
SEMENTES CERTIFICADAS & & 62,9 \\
\hline Utiliza & 22 & 31,4 \\
Não utiliza & 11 & 5,7 \\
Algumas vezes & 2 & 100,0 \\
\hline Total & 35 &
\end{tabular}

\footnotetext{
${ }^{11}$ Entre os meses de Março e Julho.

${ }^{12}$ A partir de Setembro.
}

Sociedade e Território - Natal. Vol. 29, N. 2, p. 100-114, Jul./Dez. de 2017. 
Tabela 2: Satisfação dos agricultores do PA Malehice e do PA Chaimite pelo uso de sementes certificadas

\begin{tabular}{lcc} 
SATISFAÇÃO PELO USO DE & FREQUÊNCIA & PERCENTAGEM \\
SEMENTES CERTIFICADAS & 14 & 40,0 \\
\hline Satisfeito & 3 & 8,6 \\
Não satisfeito & 7 & 20,0 \\
Depende da chuva & 11 & 31,4 \\
Não respondeu & 35 & 100,0 \\
\hline Total & & \\
\hline
\end{tabular}

Paralelamente ao baixo nível de uso de sementes certificadas, constatou-se que apenas 14,3\% dos agricultores entrevistados receberam treinamento sobre a produção de hortícolas utilizando sementes certificadas, o que pode ser considerado muito pouco. Pouco mais de metade dos agricultores $(54,3 \%)$ defendem que o aumento no uso de sementes melhoradas depende da possibilidade de serem apoiados na aquisição deste tipo de sementes, por serem de custo elevado, chegando a custar equivalente a $\mathrm{R} \$ 200$ por 100 gramas de semente. Por outro lado, referiram-se a necessidade de existência de um técnico extensionista que possa ajudar no acompanhamento do processo.

Os agricultores estão cientes e unânimes em relação a importância da intervenção do estado no suprimento de sementes e assistência técnica, contudo, em 2 anos, apenas $14,3 \%$ afirmam ter recebido o referido apoio, estando subdivididos recetores $3 \mathrm{~kg}$ de sementes certificadas de milho em um ano $(2,9 \%)$ e apoio técnico dos Serviços de Extensão Agrícola. Contudo a fraca organização dos agricultores em associações (11,43\% associados) limita a cobertura dos técnicos a mais agricultores.

Independentemente do tipo de sementes utilizadas no processo produtivo, $37,14 \%$ dos agricultores produzem para consumo familiar. Além do auto consumo, 36,3\% dos inqueridos comercializam parte da sua produção, principalmente no mercado local, como demonstrado por meio dos dados apresentados na tabela 03 .

Tabela 3: Agricultores do PA Malehice e do PA Chaimite associados e comercialização dos excedentes

\begin{tabular}{ccc} 
ASSOCIATIVISMO E & COMERCIALIZAÇÃO & ASSOCIATIVIDADE \\
COMERCIALIZAÇÃO DOS & DOS EXCEDENTES $(\%)$ & $(\%)$ \\
EXCEDENTES & & \\
\hline
\end{tabular}

Sociedade e Território - Natal. Vol. 29, N. 2, p. 100-114, Jul./Dez. de 2017. 


\begin{tabular}{lll}
\hline Sim & 31,42 & 11,43 \\
Não & 37,14 & 57,14 \\
Não respondeu & 31,43 & 31,43 \\
\hline Total & 100,0 & 100,0
\end{tabular}

\section{DISCUSSÃO}

A divisão das tarefas em função do sexo é comumente verificada nas zonas rurais em Moçambique, pois, os homens geralmente migraram do meio rural para o urbano a procura de trabalho assalariado, o que explica a predominância de mulheres na atividade agrícola nas zonas rurais (DE BRAUW, MUELLER \& LEE, 2014; YABIKU, AGADJANIAN \& CAU, 2012). Essa dinâmica populacional contribui negativamente no desenvolvimento econômico rural, através da fuga da mão-de-obra local, principalmente dos jovens que não vê na agricultura, uma atividade atraente (BEZU \& HOLDEN, 2014; PRAYITNO et al., 2013).

A dinâmica produtiva, fortemente relacionada a produtividade do fator trabalho e a adopção de tecnologias, estão diretamente relacionadas ao nível de instrução dos profissionais (CARTER, CHENG \& SARRIS, 2016; NOLTZE, SCHWARZE \& QAIM, 2012). Assim, o fato de maior parte dos agricultores inqueridos neste estudo serem pouco ou não escolarizados, pode contribuir para a reduzida taxa de adoção de sementes melhoradas. Importa aqui referir que maior parte dos extensionistas agrícolas que atuam em Moçambique não tem formação superior, havendo muitos casos de profissionais habilitados com o nível básico do ensino secundário, o que por si constitui enorme desvantagem em relação a qualidade de assistência e treinamento aos agricultores.

O fato de maior parte dos agricultores produzirem em áreas não superiores a 1 ha, aliado ao fraco investimento nesta atividade, resulta em baixa produção e produtividade, direcionada maioritariamente para o sustento familiar (DUCROT, 2016; GRAEUB et al., 2016).

Das razões apontadas pelos agricultores do setor familiar como limitantes para a exploração de áreas inferiores a 1 ha, constam a irregularidade das chuvas, frequência de secas e cheias, e a falta de recursos financeiros, factores que também foram mencionados em vários trabalhos (SIDERIUS et al., 2016; COETZEE, VAN NIEKERK \& RAJU, 2016; HARRIS \& ORR, 2014; MARVIN et al., 2013).

A dependência das chuvas para a irrigação das culturas define o período de realização do plantio. A variabilidade encontrada neste estudo, se deve em grande medida a incerteza quanto ao período de ocorrência das primeiras chuvas, dado que os agricultores do setor Sociedade e Território - Natal. Vol. 29, N. 2, p. 100-114, Jul./Dez. de 2017. 
familiar em Moçambique praticam agricultura de sequeiro (BUSSMANN et al., 2016; PIGGIN et al., 2015; TSEGAY et al., 2015; ARAYA et al., 2012).

Alguns agricultores preferem produzir culturas como o tomate entre os meses de Setembro e Dezembro, por poderem colher em períodos de escasses de produto no mercado, o que permite obter lucros elevados devido aos preços altos praticados. Contudo, verificam perdas elevadas devido a incidencia de pragas, doencas e por vezes a seca.

O papel dos Serviços de Extensão Rural é fundamental na disseminação de pacotes tecnológicos (BALOCH \& THAPA, 2016; LABARTHE \& LAURENT, 2013; BUADI, ANAMAN \& KWARTENG, 2013). Para o caso das sementes certificadas, em Chibuto, os agricultores são assistidos por extensionistas, que além de treiná-los, fazem o acompanhamento do processo produtivo, sendo solicitados pelos agricultores sempre que for necessário. No entanto, a insuficiência de extensionistas aliada a precárias condições de trabalho e a fraca organização dos agricultores, não permitem que número considerável de beneficiários seja abrangido, reduzindo a adoção e o conhecimento dos procedimentos necessários para determinada tecnologia disseminada.

Contudo, quando questionados sobre as práticas realizadas durante a produção de hortícolas utilizando-se sementes melhoradas, constatou-se entre outros, a queima de restos de plantas existentes na área de produção, durante a preparação do solo, o que poderia ser evitado, de forma a conferir maior estabilidade ao solo (COWAN, SMITH \& FITZGERALD, 2016; ABDALLA et al., 2016; LEÓN et al., 2015; SHAKESBY et al., 2015; BADÍA-VILLAS et al., 2014; TURRIÓN et al., 2012).

Os resultados deste estudo referentes a dinâmica do uso de sementes certificadas devido aos elevados preços e a insuficiência de recursos para a sua aquisição, são similares aos apresentados em vários trabalhos (ABEBE et al., 2013; MARIANO, VILLANO \& FLEMING, 2012). Entretanto, outros autores apontam para outras razões concorrentes para a não adopção deste tipo de sementes, nomeadamente, a necessidade de aquisição das sementes para cada campanha agrícola, e para o caso dos transgénicos, os potenciais riscos a biodiversidade, a saúde humana, e a incapacidade do agricultor produzir sua própria semente devendo depender do revendedor (FINCH et al., 2014; JHANSI, RANI \& USHA, 2013).

Olhando para as vantagens da adopção desta tecnologia, importa mencionar os altos rendimentos, maior tolerância a pragas e doenças, e aos fatores climáticos limitantes, menor ciclo das culturas e elevada qualidade dos produtos colhidos em comparação com as 
variedades tradicionais (DERWISCH et al., 2016; RICKER-GILBERT \& JONES, 2015). Contudo, tais vantagens não reúnem consenso entre os agricultores de Chibuto.

Os resultados satisfatórios observados pelos agricultores que utilizam sementes certificadas encorajam o aumento de esforços visando a disseminação deste tipo de material de propagação vegetal. Paralelamente, o acesso a irrigação, a assistência técnica, e ao mercado, também constituem desafios para o aumento da produção e produtividade. Contudo, é importante que a organização dos agricultores em associações seja melhorada de forma a facilitar o acesso aos serviços prestados pelas organizações públicas e privadas.

\section{CONCLUSÕES}

O nível de adoção e uso de sementes certificadas nos Postos Administrativos de Malehice e de Chaimite é baixo o que pode concorrer para a baixa produção e produtividade agrícola. Intervenções na organização dos agricultores em associações e em treinamentos e melhoria das condições de trabalho para extensionistas e para agricultores são necessárias.

\section{REFERÊNCIAS}

ABATE, Gashaw Tadesse et al. Rural Finance and Agricultural Technology Adoption in Ethiopia: Does the Institutional Design of Lending Organizations Matter? World Development, v.84, p. 235-53, 2016.

ABDALLA, Khatab et al. 2016. Long-Term Annual Burning of Grassland Increases CO2 Emissions from Soils. Geoderma, v. 282, p.80-86, 2016.

ABEBE, Gumataw K. et al. Adoption of Improved Potato Varieties in Ethiopia: The Role of Agricultural Knowledge and Innovation System and Smallholder Farmers' Quality Assessment. Agricultural Systems, v.122, p.22-32, 2013.

ARAYA, A. et al. Risk Assessment by Sowing Date for Barley (Hordeum Vulgare) in Northern Ethiopia. Agricultural and Forest Meteorology. v.154, p. 30-37, 2012.

BADÍA-VILLAS, David et al. Changes in Water Repellency, Aggregation and Organic Matter of a Mollic Horizon Burned in Laboratory: Soil Depth Affected by Fire. Geoderma, v. 213, p. 400-407, 2014.

BALOCH, Mumtaz Ali, and Gopal Bahadur Thapa. The Effect of Agricultural Extension Services: Date Farmers' Case in Balochistan, Pakistan. Journal of the Saudi Society of Agricultural Sciences, 2016.

BEYENE, Abebe D., and Menale Kassie. 2015. Speed of Adoption of Improved Maize Varieties in Tanzania: An Application of Duration Analysis. Technological Forecasting and Social Change 96, p. 298-307, 2015.

Sociedade e Território - Natal. Vol. 29, N. 2, p. 100-114, Jul./Dez. de 2017. 
BEZU, Sosina, and Stein Holden. Are Rural Youth in Ethiopia Abandoning Agriculture? World Development , v. 64: 259-72, 2014.

BEZU, Sosina, Girma T. Kassie, Bekele Shiferaw, and Jacob Ricker-Gilbert. Impact of Improved Maize Adoption on Welfare of Farm Households in Malawi: A Panel Data Analysis. World Development, v. 59, p. 120-31, 2014.

BR. 2013. “De 10 de Abril.” In I SÉRIE - NÚMERO 29, 216-40.

DE BRAUW, Alan, Valerie Mueller, and Hak Lim Lee. The Role of Rural-Urban Migration in the Structural Transformation of Sub-Saharan Africa. World Development, v. 63, p. 33$42,2014$.

BUADI, Donus K., Kwabena A. Anaman, and Joseph A. Kwarteng. Farmers' Perceptions of the Quality of Extension Services Provided by Non-Governmental Organisations in Two Municipalities in the Central Region of Ghana. Agricultural Systems, v. 120, p.20-26, 2013.

BUSSMANN, Aline, Nadir Ahmed Elagib, Manar Fayyad, and Lars Ribbe. Sowing Date Determinants for Sahelian Rainfed Agriculture in the Context of Agricultural Policies and Water Management. Land Use Policy, v. 52, p.316-28, 2016.

CABRAL, Lídia; FAVARETO, Arilson; Langton Mukwereza, and Kojo S. Amanor. Brazil's Agricultural Politics in Africa: More Food International and the Disputed Meanings of 'Family Farming. World Development , v. 81, p. 47-60, 2016.

CARTER, Michael R., Lan Cheng, and Alexandros Sarris. Where and How Index Insurance Can Boost the Adoption of Improved Agricultural Technologies. Journal of Development Economics, v. 118, p. 59-71, 2016.

COETZEE, Christo, Dewald Van Niekerk, and Emmanuel Raju. Emergent System Behaviour as a Tool for Understanding Disaster Resilience: The Case of Southern African Subsistence Agriculture. International Journal of Disaster Risk Reduction, v. 16, p.115-22, 2016.

COWAN, Ariel D., Jane E. Smith, and Stephen A. Fitzgerald. Recovering Lost Ground: Effects of Soil Burn Intensity on Nutrients and Ectomycorrhiza Communities of Ponderosa Pine Seedlings. Forest Ecology and Management, v. 378, p. 160-72, 2016.

CUNGUARA, Benedito, and Ika Darnhofer. "Assessing the Impact of Improved Agricultural Technologies on Household Income in Rural Mozambique.” Food Policy, v. 36, n. 3, p. 378390, 2011.

CUNGUARA, Benedito, and Brendan Kelly. The Impact of the PARPA II in Promoting the Agricultural Sector in Rural. (April), p. 1-27, 2015.

DERWISCH, Sebastian, Pierguiseppe Morone, Katharine Tröger, and Birgit Kopainsky. Investigating the Drivers of Innovation Diffusion in a Low Income Country Context. The Case of Adoption of Improved Maize Seed in Malawi. Futures, 2015.

DUCROT, Raphaelle Is Small-Scale Irrigation an Efficient pro-Poor Strategy in the Upper Limpopo Basin in Mozambique? Physics and Chemistry of the Earth, Parts A/B/C, 2016.

Sociedade e Território - Natal. Vol. 29, N. 2, p. 100-114, Jul./Dez. de 2017. 
FINCH, H.J.S. et al. "12 - Plant Breeding and Seed Production.” In Lockhart \& Wiseman's Crop Husbandry Including Grassland, 263-283. 2014

GRAEUB, Benjamin E. et al. The State of Family Farms in the World. World Development, v. 87 , p. $1-15,2016$.

GUANZIROLI, Carlos E., Tomás Guanziroli, Carlos E. Guanziroli, and Tomás Guanziroli. Modernização Da Agricultura Em Moçambique: Determinantes Da Renda Agrícola. Revista de Economia e Sociologia Rural, v. 53(suppl 1), p. 115-28. 2015.

HARRIS, David, and Alastair Orr. Is Rainfed Agriculture Really a Pathway from Poverty? Agricultural Systems, v. 123, p. 84-96, 2014.

JHANSI RANI, S., and R. Usha. Transgenic Plants: Types, Benefits, Public Concerns and Future. Journal of Pharmacy Research, v. 6, n. 8, p. 879-83, 2013.

LABARTHE, Pierre, and Catherine Laurent. Privatization of Agricultural Extension Services in the EU: Towards a Lack of Adequate Knowledge for Small-Scale Farms? Food Policy, p. 38 , p. 240-52, 2013.

LEÓN, Javier, M.T. Echeverría, C. Martí, and D. Badía. Can Ash Control Infiltration Rate after Burning? An Example in Burned Calcareous and Gypseous Soils in the Ebro Basin (NE Spain). Catena, v. 135, p. 377-82, 2015.

MAE. 2005. Perfil Do Distrito de Chibuto - Provincia de Gaza. 2005th ed. ed. Ministério da Administração Estatal. Chibuto: Governo de Moçambique, 2005.

MARIANO, Marc Jim, Renato Villano, and Euan Fleming. Factors Influencing Farmers' Adoption of Modern Rice Technologies and Good Management Practices in the Philippines. Agricultural Systems, v. 110, p. 41-53, 2012.

MARVIN, Hans J.P. et al. Proactive Systems for Early Warning of Potential Impacts of Natural Disasters on Food Safety: Climate-Change-Induced Extreme Events as Case in Point. Food Control, v.34, n. 2, p. 444-56, 2013.

MASSINGUE, J. et al. Ministério de Agricultura e Direcção de Economia, 2001.

MATHENGE, Mary K., Melinda Smale, and John Olwande. The Impacts of Hybrid Maize Seed on the Welfare of Farming Households in Kenya. Food Policy, v. 44, p. 262-71, 2014

MICHEELS, Eric T., and James F. Nolan. Examining the Effects of Absorptive Capacity and Social Capital on the Adoption of Agricultural Innovations: A Canadian Prairie Case Study. Agricultural Systems, v. 145, p. 127-38, 2016.

MOSCA, João. Agricultura Familiar Em Moçambique: ideologias e políticas. CEsA Working Paper, v, 127, n.34, 2014.

NOLTZE, Martin, Stefan Schwarze, and Matin Qaim. Understanding the Adoption of System Technologies in Smallholder Agriculture: The System of Rice Intensification (SRI) in Timor Leste. Agricultural Systems, v. 108, p. 64-73, 2012. 
PIGGIN, Colin et al. Effects of Tillage and Time of Sowing on Bread Wheat, Chickpea, Barley and Lentil Grown in Rotation in Rainfed Systems in Syria. Field Crops Research, v. 173, p. 57-67, 2015.

PRAKASH, Mihir. Perfil Do Sector Participatory Slum Upgrading Programme in African, Caribbean and Pacific Countries, 2011.

PRAYITNO, Gunawan, Arya Adhi Nugraha, Nindya Sari, and P.U. Yunisa Balqis. The Impact of International Migrant Workers on Rural Labour Availability (Case Study Ganjaran Village, Malang Regency). Procedia Environmental Sciences, v. 17, p. 992-98, 2013.

RICKER-GILBERT, Jacob, and Michael Jones. Does Storage Technology Affect Adoption of Improved Maize Varieties in Africa? Insights from Malawi's Input Subsidy Program. Food Policy, v. 50, p. 92-105, 2015.

SHAKESBY, Richard A. et al. Impacts of Prescribed Fire on Soil Loss and Soil Quality: An Assessment Based on an Experimentally-Burned Catchment in Central Portugal. Catena, v. 128, p. 278-93, 2015.

SIDERIUS, C. et al. The Role of Rainfed Agriculture in Securing Food Production in the Nile Basin.” Environmental Science \& Policy, v. 6, p. 14-23, 2016.

TSEGAY, Alemtsehay et al. Sowing and Irrigation Strategies for Improving Rainfed Tef (Eragrostis Tef (Zucc.) Trotter) Production in the Water Scarce Tigray Region, Ethiopia. Agricultural Water Management , v. 150, p. 81-91, 2015.

TURRIÓN, M.B. et al. Effects on Soil Organic Matter Mineralization and Microbiological Properties of Applying Compost to Burned and Unburned Soils. Journal of Environmental Management, v. 95, p. 245-49, 2012.

UAIENE, Rafael N. Moçambique: Direcção De Formação , Documentação E Transferência De Tecnologias Série De Relatórios De Pesquisa. (2). 2006

WHITFIELD, Stephen, Jami L. Dixon, Brian P Mulenga, and Hambulo Ngoma. Cerrado Meets Savannah, Family Farmers Meet Peasants: The Political Economy of Brazil's Agricultural Cooperation with Mozambique. Food Policy, v. 58, p. 70-81, 2016.

YABIKU, Scott T., Victor Agadjanian, and Boaventura Cau. Labor Migration and Child Mortality in Mozambique. Social Science e Medicine, v. 75, n.12, p. 2530-38, 2012.

YAMANO, Takashi et al. Adoption and Impacts of International Rice Research Technologies. Global Food Security, v. 8, p. 1-8, 2016.

ZANELLA, Matheus A., and Carolina Milhorance. Cerrado Meets Savannah, Family Farmers Meet Peasants: The Political Economy of Brazil's Agricultural Cooperation with Mozambique. Food Policy, v. 58, p. 70-81, 2016. 
Recebido em Setembro de 2017

Aprovado em Dezembro de 2017

Publicado em Dezembro de 2017

Sociedade e Território - Natal. Vol. 29, N. 2, p. 100-114, Jul./Dez. de 2017. 